\title{
Erratum to: Bound Entanglement for Bipartite and Tripartite Quantum Systems
}

\author{
Hui Zhao ${ }^{1}$. Sha Guo ${ }^{1}$
}

Published online: 28 May 2015

(C) Springer Science+Business Media New York 2015

\section{Erratum to: Int J Theor Phys (2015) \\ DOI 10.1007/s10773-015-2563-9}

The Journal has been informed of errors in the article named Bound Entanglement for Bipartite and Tripartite Quantum Systems by Hui Zhao and Sha Guo.

(1) The error occur in Section 2, the entries $C_{k 1}^{\dagger}$ and $C_{k 2}^{\dagger}$ of the matrixes

$$
\begin{aligned}
& \sigma_{0}=\left(\begin{array}{cccc}
C_{11} & C_{12} & \cdots & C_{1 k} \\
C_{12}^{\dagger} & C_{22} & \cdots & C_{2 k} \\
\vdots & \vdots & \ddots & \vdots \\
C_{k 1}^{\dagger} & C_{k 2}^{\dagger} & \cdots & C_{k k}
\end{array}\right) \text { and } \\
& \sigma=\varepsilon\left(\begin{array}{cccc}
C_{11} & C_{12} & \cdots & C_{1 k} \\
C_{12}^{\dagger} & C_{22} & \cdots & C_{2 k} \\
\vdots & \vdots & \ddots & \vdots \\
C_{k 1}^{\dagger} & C_{k 2}^{\dagger} & \cdots & C_{k k}
\end{array}\right)+(1-\varepsilon)\left(\begin{array}{ccc}
D_{11} & \cdots & 0 \\
\vdots & \ddots & \vdots \\
0 & \cdots & D_{k k}
\end{array}\right) \text { should be } C_{1 k}^{\dagger} \text { and } C_{2 k}^{\dagger} .
\end{aligned}
$$

The online version of the original article can be found at http://dx.doi.org/10.1007/s10773-015-2563-9.

Hui Zhao

zhaohui@bjut.edu.cn

1 College of Applied Science, Beijing University of Technology, Beijing 100124, China 
(2) The error occur in Section 3, the matrix $E_{11}=\left(\begin{array}{cccccccc}E_{1} & 0 & E_{2} & 0 & E_{3} & 0 & 0 & 0 \\ 0 & 0 & 0 & 0 & 0 & 0 & 0 & 0 \\ E_{2}^{\dagger} & 0 & E_{1} & 0 & 0 & 0 & E_{4} & 0 \\ 0 & 0 & 0 & 0 & 0 & 0 & 0 & 0 \\ E_{3}^{\dagger} & 0 & 0 & 0 & E_{5} & 0 & E_{6} & 0 \\ 0 & 0 & 0 & 0 & 0 & 0 & 0 & 0 \\ 0 & 0 & E_{4}^{\dagger} & 0 & E_{6}^{\dagger} & 0 & E_{1} & 0 \\ 0 & 0 & 0 & 0 & 0 & 0 & 0 & 0\end{array}\right)$ should be

$$
E_{11}=\left(\begin{array}{cccccccc}
E_{1} & 0 & E_{2} & 0 & E_{3} & 0 & 0 & 0 \\
0 & 0 & 0 & 0 & 0 & 0 & 0 & 0 \\
E_{2}^{\dagger} & 0 & E_{7} & 0 & 0 & 0 & E_{4} & 0 \\
0 & 0 & 0 & 0 & 0 & 0 & 0 & 0 \\
E_{3}^{\dagger} & 0 & 0 & 0 & E_{5} & 0 & E_{6} & 0 \\
0 & 0 & 0 & 0 & 0 & 0 & 0 & 0 \\
0 & 0 & E_{4}^{\dagger} & 0 & E_{6}^{\dagger} & 0 & E_{8} & 0 \\
0 & 0 & 0 & 0 & 0 & 0 & 0 & 0
\end{array}\right)
$$

where the entries $E_{7}=-\left(P_{12} E_{2}+E_{4} P_{24}\right)$ and $E_{8}=-\left(P_{24} E_{4}+P_{34} E_{6}\right)$.

(3) The error occur in equation (36)

$$
\left(r_{1}, r_{2}, r_{3}, r_{4}\right)^{t} \otimes\left(s_{1}, 0, s_{3}, 0,0, s_{6}, 0, s_{8}, s_{9}, 0, s_{11}, 0, s_{13}, 0, s_{15}, 0\right)^{t}
$$

should be

$$
\left(r_{1}, r_{2}, r_{3}, r_{4}\right)^{t} \otimes\left(s_{1}, 0, s_{3}, 0, s_{5}, 0, s_{7}, 0,0, s_{10}, 0, s_{12}, 0, s_{14}, 0, s_{16}\right)^{t} .
$$

(4) The error occur in equation (37), the vectors

$$
\begin{aligned}
& \left(c_{1}, c_{2}, 0,0,0,0,0,0, c_{9}, c_{10}, 0,0,0,0,0,0\right)^{t} \otimes\left(d_{1}, d_{2}, d_{3}, 0\right)^{t}, \\
& \left(0,0, c_{3}, c_{4}, 0,0,0,0,0,0, c_{11}, c_{12}, 0,0,0,0\right)^{t} \otimes\left(d_{1}, d_{2}, 0, d_{4}\right)^{t}, \\
& \left(0,0,0,0, c_{5}, c_{6}, 0,0,0,0,0,0, c_{13}, c_{14}, 0,0\right)^{t} \otimes\left(d_{1}, 0, d_{3}, d_{4}\right)^{t}, \\
& \left(0,0,0,0,0,0, c_{7}, c_{8}, 0,0,0,0,0,0, c_{15}, c_{16}\right)^{t} \otimes\left(0, d_{2}, d_{3}, d_{4}\right)^{t},
\end{aligned}
$$

should be

$$
\begin{aligned}
& \left(0, c_{2}, 0,0,0,0,0,0, c_{9}, 0,0,0,0,0,0,0\right)^{t} \otimes\left(d_{1}, d_{2}, d_{3}, 0\right)^{t}, \\
& \left(0,0,0, c_{4}, 0,0,0,0,0,0, c_{11}, 0,0,0,0,0\right)^{t} \otimes\left(d_{1}, d_{2}, 0, d_{4}\right)^{t}, \\
& \left(0,0,0,0,0, c_{6}, 0,0,0,0,0,0, c_{13}, 0,0,0\right)^{t} \otimes\left(d_{1}, 0, d_{3}, d_{4}\right)^{t}, \\
& \left(0,0,0,0,0,0,0, c_{8}, 0,0,0,0,0,0, c_{15}, 0\right)^{t} \otimes\left(0, d_{2}, d_{3}, d_{4}\right)^{t} .
\end{aligned}
$$

The authors regret these errors. 